\title{
Dynamic transition in an atomic glass former: a molecular dynamics evidence
}

\author{
Estelle Pitard, ${ }^{1}$ Vivien Lecomte, ${ }^{2}$ and Frédéric van Wijland ${ }^{3}$ \\ ${ }^{1}$ Laboratoire des Collö̈des, Verres et NanoMatériaux (CNRS UMR 5587), \\ Université de Montpellier II, place Eugène Bataillon, 34095 Montpellier cedex 5, France \\ ${ }^{2}$ Laboratoire de Probabilités et Modèles Aléatoires (CNRS UMR 7599), \\ Université Paris Diderot - Paris 7 et Université Pierre et Marie Curie - Paris 6, \\ Site Chevaleret, CC 7012, 75205 Paris cedex 13, France \\ ${ }^{3}$ Laboratoire Matière et Systèmes Complexes (CNRS UMR 7057), Université Paris Diderot - Paris 7, \\ 10 rue Alice Domon et Léonie Duquet, 75205 Paris cedex 13, France
}

\begin{abstract}
We find that a Lennard-Jones mixture displays a dynamic phase transition between an active regime and an inactive one. By means of molecular dynamics simulations and of a finite-size study, we show that the space time dynamics in the supercooled regime coincides with a dynamic first order transition point.
\end{abstract}

In order to realize that a physical system has fallen into a glassy state one must either drive the system out of equilibrium, or investigate its relaxation properties. It is indeed a well-known fact that since no static signature is available, one has to resort to nonequilibrium protocols or measurements to identify a glassy state. The supercooled regime, which sets in before the material actually freezes into a solid glass, is also characterized by anomalous temporal properties, such as the increase in the viscosity, and ageing phenomena during relaxation processes. The density-density autocorrelation function at a microscopic scale, instead of exhibiting a straightforward exponential relaxation, develops a plateau (over a duration conventionally called $\tau_{\alpha}$ ) before relaxing to zero. The present work is concerned with the dynamical properties in the supercooled regime. The idea that dynamical heterogeneities, long-lived large scale spatial structures, are the trademark of the slow and intermittent dynamics in the supercooled regime dates back to almost thirty years [1] and many developments have occurred since [2 [4]. Here, instead of resorting to local probes such as the van Hove function, the self-intermediate scattering function, or the nonlinear dynamical susceptibility, - i.e on multi-point correlation functions in space and time-, we prefer to rely on a global characterization of the system's dynamics.

More recently, it was advocated by Garrahan et al. [5] that, at the level of Kinetically Constrained Models (KCM), dynamical heterogeneities appeared as the consequence of a universal phase transition mechanism, largely independent of the specific model under consideration. The phase transitions at work are not of a conventional type: they occur in the space of trajectories of realizations the system has followed over a large time duration, instead of occurring in the space of available configurations, as the liquid-gas transition, or a putative thermodynamic glass transition, for instance, do. There exists a well-established body of mathematical physics literature to analyze these phase transitions, which is based, in spirit, on the thermodynamic formalism of Ruelle [6, 7], and which was adapted [8] and then exploited [5] in numerical and analytical studies of the
KCM's. The idea behind these studies is to follow the statistics, upon sampling the physical realizations of the system over a large time duration, of a space and time extensive observable. This observable characterizes the level of dynamical activity in the course of the system's time evolution. At this stage, the term activity refers to the intuitive picture that an active trajectory is characterized by many collective rearrangements needed to escape from energy basins, whereas an inactive trajectory is dominated by the rapid in-cage rattling motion of the particles without collective rearrangements. This activity observable is used to partition trajectories into two groups - the inactive and the active ones- depending on whether their activity is above or below the average activity.

An important step forward in probing the relevance of the dynamic phase transition scenario to realistic glasses is the work of Hedges et al. [9] in which an atomistic model was considered. The system studied by these authors is a mixture of two species of particles interacting via a Lennard-Jones potential otherwise known as the Kob-Andersen (KA) model [10], and which has been shown to fall easily into a glassy state without crystallizing. Endowed with a Monte-Carlo or a Newtonian dynamics, Hedges et al. implemented the Transition Path Sampling (TPS) method to produce the probability distribution function (pdf) of the activity, at finite times. These authors succeeded in showing that for a finite system, as the observation time was increased, the pdf of the activity develops a bimodal structure. In the light of previous works on lattice models for glass formers, they interpreted their result as an evidence for a bona fide dynamic phase transition, expected to occur once the infinite size and infinite observation time limits are reached. While the work of Hedges et al. is a definite breakthrough towards a better understanding of atomistic glass formers, it leaves a number of questions unanswered. These are the subject of the present work. Dynamic phase transitions occur in the large time limit, as exemplified by the pioneering works of Ott et al. [11], Cvitanović [12 or Szépfalusy and Tél [13] (see Beck and Schlögl [14] for further references). The dynamic 
transitions at work in glass formers are of a subtler form, since they only emerge upon, in addition, considering the infinite system size limit.

Our first goal in this letter is to show the existence of a phase transition. Our second goal is to be more precise about the location of the phase transition itself. This is indeed a central issue: if the critical point is away from the typical measurable activity then the transition scenario is at best a crossover. If, on the contrary, it is shown that the phase transition actually takes place for typical trajectories then experimentally observable consequences are expected. Our third goal is to provide a rigorous definition of the activity. It is our feeling that deeper insight will be gained if the notion of activity can be characterized by standard physical concepts (like forces between particles) rather than on phenomenological considerations.

In the present work, we consider a KA mixture, which we study by extending the methods of Molecular Dynamics (MD) to the study of temporal large deviations. By implementing a MD version of the cloning algorithm of Giardinà, Kurchan and Peliti 15] we are able to follow the statistics of the large deviations of the activity. Our activity observable is a physically transparent quantity which can be related to the rate at which the system escapes from a given location in phase space. As we shall soon see, the typical trajectories of the system in phase space are characterized by strong space time heterogeneities -the so-called dynamical heterogeneitieswhich appear to be the by-product of an underlying first order dynamical transition in which the activity plays the role of an order parameter. A typical realization lies at a first-order transition point, which is thus characterized by the coexistence of competing families of trajectories. Active trajectories, in which a time and space extensive number of events where a particle escapes from its local cage, coexist with inactive trajectories in which localization of the particles within their local neighborhood dominates the dynamics. Without going further into the mathematical details of what the activity is, we present in figure 1 a snapshot of a the activity map in a threedimensional KA mixture in three distinct situations.

We now carry out our program and we begin by defining an activity observable: given a set of particles interacting via a two-body potential $V\left(\mathbf{r}_{i}-\mathbf{r}_{j}\right)$, in which each particle $i$ is subjected to a force $\mathbf{F}_{i}=-\sum_{j \neq i} \nabla V\left(\mathbf{r}_{i}-\right.$ $\mathbf{r}_{j}$ ), we introduce the observable:

$$
V_{\mathrm{eff}}=\sum_{i}\left[\frac{\beta}{4} \mathbf{F}_{i}^{2}+\frac{1}{2} \boldsymbol{\nabla}_{\mathbf{r}_{i}} \cdot \mathbf{F}_{i}\right]
$$

The combination $\frac{\beta}{4} \mathbf{F}_{i}^{2}+\frac{1}{2} \boldsymbol{\nabla}_{\mathbf{r}_{i}} \cdot \mathbf{F}_{i}$ can be interpreted as the activity of a single particle. The quantity $V_{\text {eff }}$ in (1) appears in the study of Brownian interacting particles and measures the tendency for dynamical trajectories to evolve away from a given configuration. Indeed, it can
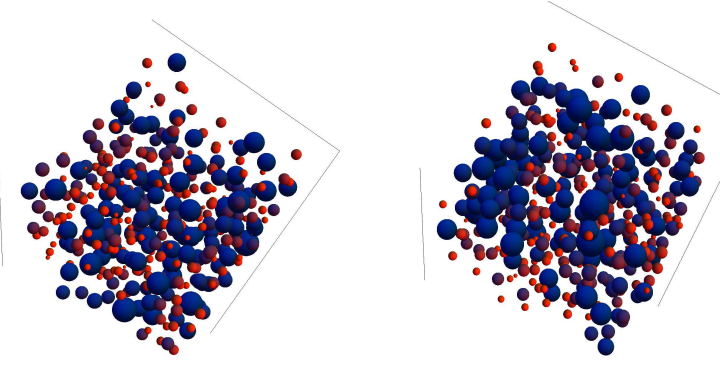

FIG. 1: In these snapshots, the diameter of the particles quantifies the local activity: small points refer to mobile active particles, whereas large blobs refer to blocked inactive particles. Red (blue) indicates that the activity is larger (smaller) than the median one. Left: Activity map in a typical configuration from a trajectory with excess activity with respect to the average activity; there is a large number of mobile particles though blocked particles also exist. Right: Activity map in a typical configuration from a trajectory with less activity than the average activity, large blobs dominate.

be argued [16] that the quantity $\exp \left(-\beta V_{\text {eff }} \mathrm{d} t\right)$ is proportional to the probability $P(x, t+d t \mid x, t)$ that the system has stayed in its configuration between $t$ and $t+\mathrm{d} t$. This means that $\beta\left|V_{\text {eff }}\right|$ is the rate at which the system escapes its configuration. To understand how (1) is related to this escape rate one writes $P(x, t+d t \mid x, t)=$ $\left\langle x\left|e^{-\hat{H} \mathrm{~d} t}\right| x\right\rangle$ where $\hat{H}$ is the Fokker-Planck operator of evolution of the system. Using the detailed balance property of the dynamics, $\hat{H}$ can be symmetrized and this leads to $P(x, t+d t \mid x, t) \sim \exp \left(-\beta V_{\text {eff }} \mathrm{d} t\right)$, using standard path-integral representation of the propagator $\left\langle x\left|e^{-\hat{H} \mathrm{~d} t}\right| x\right\rangle$ (see [16] for details). Besides, if one regularizes the Brownian dynamics in the form of hops on a lattice, our activity can be viewed as the continuum ana$\log$ of the activity introduced in Lecomte et al. 17] which counted the number of configuration changes undergone by a system over a given time interval. Perhaps more interestingly still, $V_{\text {eff }}$ also appears as the continuum limit analog of the dynamical complexity (a trajectorydependent Kolmogorov-Sinai entropy [8]) which further clarifies its conceptual value. However, hand-waving arguments lead to a more concrete understanding of the value of the effective potential $V_{\text {eff. }}$. Indeed, in the expression (11) minimizing the first term in the right hand side drives the system away from regions of phase space where forces are nonzero. In other words, it tends to favor mechanical equilibrium configurations (whether stable or metastable). The second term in the right hand side of (1), can be positive or negative: if negative, it will favor local minima, all the more so as they are deep and steep; if positive, it will select local maxima. As a result, there will be two classes of trajectories, according to the values of $V_{\text {eff. It }}$ can be shown that the average equilibrium value of $V_{\text {eff }}$ is $\left\langle V_{\text {eff }}\right\rangle=-\beta / 4 \sum_{i} F_{i}^{2}$, which is always negative. For minimal (negative) values of $V_{\text {eff }}$, trajectories will explore deep energy basins, for maximal values of $V_{\text {eff }}$, trajectories will explore local maxima of the energy landscape. 


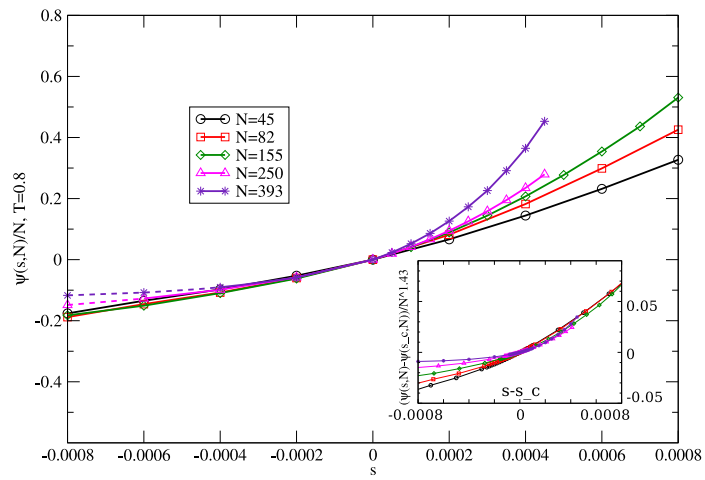

FIG. 2: The dynamical free energy per particle $\frac{\psi(s, N)}{N}$ is shown as a function of $s$ for increasing sizes $N=45,82$, 155, 250 and 393. While the curves collapse onto a single master curve at $s<0$, a strong size-dependent behavior is observed at $s>0$. On the $s>0$ side, the curves display a rapid variation at a typical value $s_{c}(N)$ which decreases as $N$ increases, leading to the putative building up of a singularity of $\psi(s, N) / N$ at $s=0$ as $N \rightarrow \infty$. The rescaled $\left[\psi(s, N)-\psi\left(s_{c}, N\right)\right] / N^{1+\alpha}$ with $\alpha \simeq 0.43$ is shown in the inset: on the inactive side $s>0$ the curves collapse onto a single curve, exemplifying the large $N$ finite size scaling. Applying the cloning algorithm requires the $\left|s V_{\text {eff }} \mathrm{d} t\right|$ product to be infinitesimally small (in principle), which at the left end of the graph and for the largest two values of $\mathrm{N}$ is hardly verified (dashed lines). For these points, this product reaches .5 which accounts for the increasing error.

The total activity is defined as $K(t)=\int_{0}^{t} \mathrm{~d} t^{\prime} V_{\text {eff }}\left(t^{\prime}\right)$. In terms of the local density $\rho(\mathbf{x}, t)$, our activity reads:

$$
\begin{aligned}
K= & \int_{0}^{t} \mathrm{~d} t\left[\frac{1}{4 T} \int_{\mathbf{x}, \mathbf{y}, \mathbf{z}} \rho(\mathbf{x}, t) \rho(\mathbf{y}, t) \rho(\mathbf{z}, t) \nabla V(\mathbf{x}-\mathbf{y}) \boldsymbol{\nabla} V(\mathbf{x}-\mathbf{z})\right. \\
& \left.-\frac{1}{2} \int_{\mathbf{x}, \mathbf{y}} \rho(\mathbf{x}, t) \rho(\mathbf{y}, t) \Delta V(\mathbf{x}-\mathbf{y})\right]
\end{aligned}
$$

It is now apparent that $V_{\text {eff }}$ involves three-body effective interactions, a fact that we a posteriori interpret by realizing that in order to escape from an energy basin via a collective rearrangement, multi-body interaction terms are needed.

Given the activity we have just defined, we set out to determine the distribution of this quantity over a large number of realizations of the process, that is, given the dynamics is deterministic, by sampling over a large number of initial states drawn from the equilibrium Boltzmann distribution. Sampling the full distribution of the activity requires sufficient statistics beyond its typical values. For that reason we have preferred to work in an ensemble of trajectories in which the average activity, rather than the activity, is fixed. In this canonical version, we consider a large number of systems evolving in parallel, and between $t$ and $t+\mathrm{d} t$ we remove or add a system in a given configuration with a cloning factor equal to $\exp \left(-s V_{\text {eff }}(t) \mathrm{d} t\right)$. How to perform a numerical simulation in a canonical ensemble of time realiza- tions was explained in Giardinà et al. [15]'s cloning algorithm. We combined the cloning algorithm with the molecular dynamics simulation of the interacting particles. Choosing a positive value of $s$ allows one to focus on time realizations with a lower-than-average activity, while a negative $s$ selects over-active trajectories, and $s$ close to zero samples typical activity trajectories. To this day, no one has been able to endow the parameter conjugate to the activity $s$ with a concrete physical meaning, so that the only physically realizable value of an $s$-ensemble is achieved for $s=0$. As will shortly become clear, analyzing the $s=0$ properties gains enormously from being able to vary $s$ away from 0 , a theorist's privilege. The distribution of the activity $K$ is fully encoded in its generating function $Z(s, t, N)=\left\langle\mathrm{e}^{-s K}\right\rangle$, or, alternatively, in the corresponding cumulant generating function $\ln Z$. We define the intensive dynamical free energy $\psi(s, N)=\lim _{t \rightarrow \infty} \frac{\ln Z}{t}$ for any given finite size. From the knowledge of the function $\psi(s, N)$ we can reconstruct the properties of the activity $K$. In numerical terms, in view of the cloning algorithm used, $\psi$ stands for the growth rate of the population of systems evolving in parallel. The details of the simulations are as follows. We used the A-B mixture of [10] for samples of different sizes. The samples all had the approximate ratio $80 / 20$ for A/B particles. They were first prepared at equilibrium at temperature $T=0.8$ by coupling to a stochastic heat bath; the time step was $\mathrm{d} t=2 \cdot 10^{-2}$. During a very long simulation at equilibrium, the $N_{c}$ clones where prepared. Then the cloning algorithm of Giardinà et al. [15] was performed with a small coupling to the heat bath, necessary to provide some stochasticity to the exploration of trajectory space, as explained in [15, 18] for deterministic systems. In our simulations, the convergence was checked by varying the number of clones and the duration of the simulations. For $N=45,82$ the best results were found for $N_{c}=1000$ and $\tau=10 \tau_{\alpha}\left(\tau_{\alpha}\right.$ is the relaxation time); for $N=155,250,393$ the best results were found for $N_{c}=500$ and $\tau=20 \tau_{\alpha}$. Fluctuations in the space of clones would have required for the largest system sizes that we substantially increase $N_{c}$ as the tails of the distribution are sampled (extreme values of $s$ ).

We now present the central result of the work, namely the dynamical free energy $\psi(s, N)$ as a function of $s$ for increasing system sizes $N$. Figure 2 shows that the dynamical free energy per particle $\frac{\psi(s, N)}{N}$ settles to a welldefined limiting value for $s<0$ as $N$ increases. This means that for $s<0$ there exists a thermodynamic limit. On the $s<0$ side, where the activity is above its average value at $s=0$, which we interpret in terms of active trajectories, the physical properties are not far from those at $s=0$ as predicted from the Gibbs equilibrium distribution. However, a dramatic change in behavior is observed for positive values of $s$. Not only do the dynamical free energies exhibit a strong size dependence, but a severe change in behavior is also observed on that side of the parameter space. For $s>0$, the free energy rapidly increases, and the activity rapidly decreases. The 


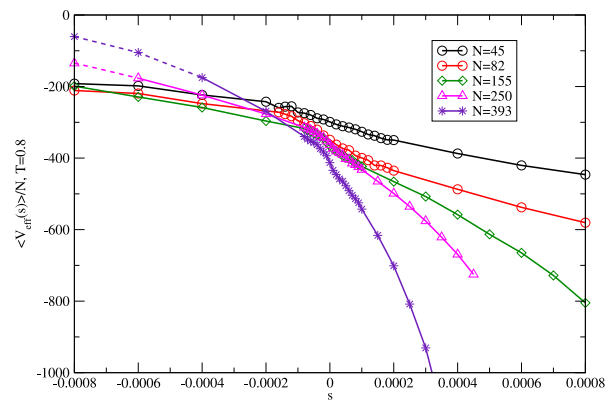

FIG. 3: The average activity per particle $\left\langle V_{\text {eff }}\right\rangle / N$ is measured in an ensemble of trajectories biased by a weight $\mathrm{e}^{-s K}$ and is plotted as a function of $s$. While the $s<0$ regime shows convergence to a size independent limit of an order comparable to its equilibrium value, the $s>0$ side confirms a strongly size-dependent behavior as $V_{\text {eff }}$ abruptly decreases to negative values. This sudden drop in $V_{\text {eff }}$ indicates that the system freezes into inactive states.

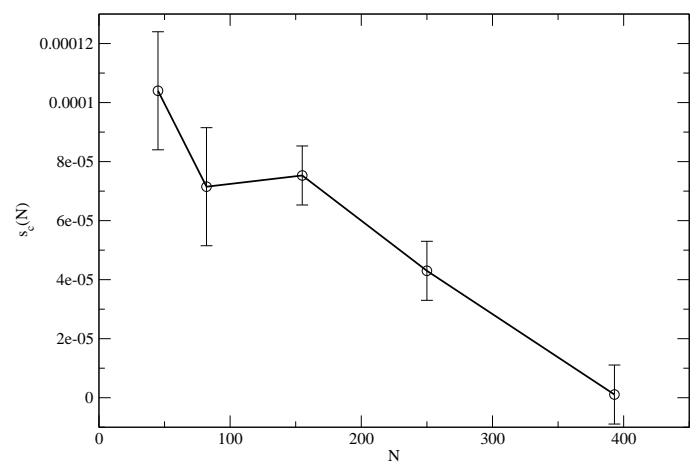

FIG. 4: $s_{c}(N)$ as a function of $N$.

location $s_{c}(N)$ at which this rapid increase sets shows a strong $N$-dependence: $s_{c}(N)$ seems to decrease to zero as $N$ increases. The precise location of $s_{c}(N)$ has been determined thanks to a spline method and by maximizing $\psi^{\prime \prime}(s)$; this is illustrated in figure 4. One notes that given the existence of error bars, there is an overall decrease of $s_{c}(N)$ as $N$ increases, but one cannot exclude that $s_{c}(N)$ converges to a very small positive value. As in other examples of first order dynamical transitions [5], we remark that $s_{c}(N)$ remains positive for all finite $N$. Indeed, $\psi^{\prime}(0)=-\left\langle V_{\text {eff }}\right\rangle$ is proportional to $N$, which means that $\psi(s)$ also scales as $N$ in the vicinity of $s=0$, and since $s_{c}(N)$ marks the transition to a regime where $\psi(s)$ scales differently with $N$, one has $s_{c}(N)>0$.

The interpretation in terms of active $v s$. inactive trajectories is easier when plotting the average activity as a function of $s$. This is done in figure 3, where the average activity is measured independently: we see that in the vicinity of $s_{c}(N)$, the behavior of $\left\langle V_{\text {eff }}\right\rangle / N$ changes abruptly from a smooth range of values at $s<0$ to a strongly negative range for $s>0$. In this inactive regime, through rescaling (see the inset on figure 21), we see that the free energy behaves in $N^{1+\alpha}$ with $\alpha \simeq 0.43$, in contrast to the purely extensive behavior of the active regime. A similar difference of scaling exponents in $N$ is observed in KCMs in two dimensions [5] at $s>0: \psi(s)$ is of order $L$ for the Fredrickson-Anderson model (FA) while of order 1 for the triangular lattice gas (TLG). This behavior is related to the geometry of the remaining active sites in the system: those divide up along the border of fully inactive domains in the FA model, while they are isolated in the TLG. Our finding that $\alpha$ is non-integer indicates that the KA mixture adopts configurations with non-trivial geometrical features for the inactive particles in the inactive phase due to the effective long-range interactions that develop in the $s>s_{c}$ states. Since large values of $\left|V_{\text {eff }}\right|$ correspond to inactive histories, it is expected that $\alpha>0$, as observed. We note that such finitesize behaviour of the activity with non-trivial exponents is known to occur in lattice KCMs [19], where the value of those exponents are directly related to the nature of configurations appearing in the inactive state (which differ from those of the active state).

This allows us to claim that there exists a phase transition from inactive to active states as $s$ is varied. Our closely related goal was to identify the location of the transition. We have shown that $s_{c}(N)$ displays a strong size-dependence with an overall decrease for the range of $N$ values explored. The two different well-defined scalings $\sim N^{1+\alpha}($ resp. $\sim N)$ for $s>0($ resp. $s<0)$ indicate that we have reached the large $N$ asymptotics on our range of system sizes (see figure 2). We therefore conclude that the most likely value at which the transition takes place in an infinite system is either $s_{c}(\infty)=0$ or a very small positive value close to 0 . We were not able to perform simulations for larger values of $N$ due to the very large computation times needed.

The first order dynamic transition scenario observed in KCM's is thus confirmed in the atomistic model we have studied. As we mentioned earlier, the location of the phase transition along the $s$-variable axis is an extremely relevant issue since only the value $s=0$ is experimentally accessible. Note however that identifying a transition point at $s=0$ is possible only on the condition that $s$ is varied across 0 . Therefore, finding evidence for the transition occurring at $s=0$ renders an experimental observation of the transition a credible achievement. The lesson to be drawn from the present extensive simulation series is that, as expected, it is desirable to work on as small systems as possible, yet large enough to allow for collective effects in trajectory space to develop.

This work was supported by a French Ministry of Foreign Affaires Alliance grant. VL was supported in part by the Swiss NSF under MaNEP and Division II. We greatly benefited from discussions with R.L. Jack. We thank W. Kob for providing the software for the preparation of the configurations, and L. Berthier for help with figure 1. 
[1] G.H. Fredrickson, H.C. Andersen, Phys. Rev. Lett. 53 1244 (1984).

[2] M.M. Hurley, P. Harrowell, J. Chem. Phys. 10510521 (1996). M.D. Ediger, Annu. Rev. Phys. Chem. 5199 (2000).

[3] J.P. Garrahan and D. Chandler, Phys. Rev. Lett. 89, 035704 (2002).

[4] M. Merolle, J.P. Garrahan and D. Chandler, Proc. Natl. Acad. Sci. USA 102, 10837 (2005); R.L. Jack, J.P. Garrahan and D. Chandler, J. Chem. Phys. 125, 184509 (2006).

[5] J.P. Garrahan, R.L. Jack,V. Lecomte, E. Pitard, K. van Duijvendijk and F. van Wijland, Phys. Rev. Lett. 98, 195702 (2007); J. Phys. A 42, 075007 (2009).

[6] D. Ruelle, Thermodynamic Formalism (Addison-Wesley, Reading, 1978).

[7] P. Gaspard, Chaos, scattering and statistical mechanics (CUP, Cambridge, 1998).

[8] V. Lecomte, C. Appert-Rolland, and F. van Wijland, Phys. Rev. Lett. 95, 010601 (2005).

[9] L. Hedges, R.L. Jack, J.-P. Garrahan, and D.C. Chandler, Science 323, 1309 (2009).
[10] W. Kob and H.C. Andersen, Phys. Rev. E 48, 4364 (1993).

[11] E. Ott, W. Withers, and J.A. Yorke, J. Stat. Phys. 36, 697 (1984).

[12] R. Artuso, P. Cvitanović, and B.G. Kenny, Phys. Rev. A 39, 268 (1989).

[13] P. Szépfalusy and T. Tél, Phys. Rev. A 35, 477 (1987).

[14] C. Beck and F. Schögl, Thermodynamics of chaotic systems, and introduction, Cambridge University Press, 1993.

[15] C. Giardinà, J. Kurchan, and L. Peliti, Phys. Rev. Lett. 96, 120603 (2006).

[16] E. Autieri, P. Faccioli, M. Sega, F. Pederiva, and H. Orland, J. Chem. Phys. 130, 064106 (2009).

[17] V. Lecomte, C. Appert-Rolland, and F. van Wijland, J. Stat. Phys. 127, 51 (2007).

[18] J. Tailleur and J. Kurchan, Nature Physics 3, 203 (2007)

[19] T. Bodineau and C. Toninelli, Activity phase transition for constrained dynamics, arXiv:1101.1760, accepted for publication in Comm. Math. Phys (2011) 